\title{
High Platelet Count
}

National Cancer Institute

\section{Source}

National Cancer Institute. High Platelet Count. NCI Thesaurus. Code C162107.

A higher than average level of platelets in a sample. 